Alexander Eisenkopf*

\title{
PPP - kein relevanter Beitrag zur Lösung der Infrastrukturprobleme in Deutschland
}

https://doi.org/10.1515/zfwp-2017-0018

Abstract: Public Private Partnerships (PPPs) are the cooperation between the state and the private sector in the planning, realization, financing and operation of traditionally public services. In the area of road infrastructure, they are implemented as F-, A- or V-models. Statements on the profitability of PPP projects are always subject to case studies. As a rule, financing costs for PPPs will be higher than for conventional procurement. Cost savings in PPP, on the other hand, are empirically relatively low, since independent and transparent studies hardly exist. Finally, there are considerable doubts as to whether PPP projects contribute to an increase in efficiency from a macroeconomic perspective and deliver a relevant contribution to the solution of infrastructure problems in Germany. This seems especially to be true with respect to the intended establishment of a federal infrastructure company for motorways.

\section{Hintergrund: Verkehrsinfrastrukturpolitik in der Krise}

In Deutschland wird seit vielen Jahren über die Unterfinanzierung und die Steuerungsprobleme bei der Bereitstellung der Verkehrsinfrastruktur diskutiert. Spätestens seit der Vorlage der Ergebnisse der sogenannten Pällmann-Kommission im Jahre 2000 ist der Begriff der Instandhaltungskrise etabliert, der den fortlaufenden Substanzverzehr der Verkehrsinfrastruktur beschreibt (Kommission Verkehrsinfrastrukturfinanzierung, 2000). Die sogenannte Daehre-Kommission machte in ihrem im Dezember 2012 vorgelegten Bericht den Versuch einer Quantifizierung dieser Instandhaltungslücke, wodurch sich im politischen Raum die Intensität der Diskussion und der Handlungsdruck weiter erhöht haben (Kommission „Zukunft der Verkehrsinfrastrukturfinanzierung“, 2012). Der sogenannte „Investitionshochlauf“ in der letzten Legislaturperiode und das Gesetzgebungsverfahren zur Infrastrukturgesellschaft sind Ausfluss dieses zunehmenden Handlungsdrucks.

*Kontakt: Alexander Eisenkopf, ZEPPELIN Universität, Lehrstuhl für Wirtschafts- und Verkehrspolitik, Am Seemoser Horn 20, 88045 Friedrichshafen, Tel.: (07541) 6009-1211, E-Mail: alexander.eisenkopf@zu.de 
Mit der Verabschiedung des neuen Bundesverkehrswegeplans $2030 \mathrm{im} \mathrm{Au-}$ gust 2016 wurde die finanzielle Dimension des Problems noch einmal bestätigt. Das Gesamtvolumen des BVWP 2030 beträgt 269,6 Mrd. €, wovon 69 \% für Erhaltung und Ersatzinvestitionen verwandt werden sollen. Trotzdem entfallen auf die geplante Investitionssumme des vordringlichen Bedarfs (inkl. Schleppe) 98,3 Mrd. € gegenüber 90,5 Mrd. € beim BVWP 2003 (BMVI, 2016). Diese Zahlen verdeutlichen, dass vor dem Hintergrund eines weiteren Verkehrswachstums hohe zusätzliche Finanzmittel erforderlich sind, um die Leistungsfähigkeit der Verkehrsinfrastruktur auf Dauer zu sichern.

Eine rationale und an Effizienzkriterien orientierte Verkehrsinfrastrukturpolitik sollte deshalb drei Prinzipien im Blick behalten. Zum einen geht es um eine an durchaus komplexen Kriterien auszurichtende bedarfsgerechte Finanzierung der Verkehrsinfrastruktur. Weiterhin sollte die Verkehrspolitik effizienzorientierten Regeln bei der Auswahl der zu realisierenden Projekte z. B. im Rahmen der Bundesverkehrswegeplanung folgen (Stichwort: Priorisierung). Rationale Verkehrspolitik muss aber drittens auch gewährleisten, dass die realisierbaren Projekte möglichst effizient gebaut und betrieben werden: Dies adressiert den institutionellen Rahmen der Bereitstellung der Verkehrsinfrastruktur. Mit dem Begriff der Public Private Partnership (PPP) sind sowohl der Finanzierungs- wie auch institutionelle Aspekte der Infrastrukturpolitik angesprochen, denn im Rahmen von PPP übernehmen private Akteure Aufgaben der Planung, Finanzierung, Bereitstellung und Bewirtschaftung von Fernstraßen. Der vorliegende Beitrag widmet sich dem Spannungsfeld herkömmlicher Organisationsund Bereitstellungsformen, institutioneller Neuerungen und privater (Mit-)Finanzierung der Bundesfernstraßen im Rahmen von PPP vor dem Hintergrund der mit der Einrichtung einer Infrastrukturgesellschaft politisch beschlossenen Neuordnung der Zuständigkeiten für die Fernstraßeninfrastruktur.

\section{PPP: Grundsätzliche Lösungsansätze und Modellstrukturen}

Zur Institutionalisierung öffentlich-privater Leistungsprozesse gilt heute das Konzept der Public Private Partnership (PPP) bzw. der öffentlich-privaten Partnerschaft (ÖPP) als State of the Art. ${ }^{1}$ Hierunter lässt sich generell die Kooperation zwischen Staat und Privatwirtschaft bei der Planung, Erstellung, Finanzierung

1 Im Folgenden wird der Terminus PPP verwendet; allerdings hat sich im deutschsprachigen Raum auch der ÖPP-Begriff mittlerweile etabliert. 
und beim Betrieb bzw. bei der Verwertung bislang ausschließlich staatlicher Leistungen verstehen. Ihr Ziel liegt insbesondere in einer effizienteren Erstellung von Leistungen, welche auf die Deckung kollektiver Bedarfe ausgerichtet sind, die in den Bereich der Daseinsvorsorge fallen (Delmon, 2011).

Wenngleich die Finanzierungsfunktion seit langer Zeit im Fokus der politischen Diskussion um PPP steht, ist sie zwar ein zentraler, aber nicht der ausschließliche Leistungsbereich einer PPP. Vielmehr verfolgen PPP typischerweise ein so genanntes Lebenszykluskonzept: Zur Erschließung möglichst hoher Effizienzpotentiale werden über die Finanzierungsfunktion hinaus weitere Aufgaben während des Projektlebenszyklus einbezogen, wobei ein umfassender Risikotransfer auf Private angestrebt wird. Die privaten Unternehmen stellen statt isoliert erbrachter Bau-, Betriebs- oder Finanzierungsleistungen umfassende Problemlösungen bereit und garantieren diese Leistungen. Charakteristisch für PPP ist daher der dauerhafte oder zumindest langfristige Leistungsverbund von öffentlicher Hand und privaten Unternehmen.

Zur Umsetzung von PPP wurden in Wissenschaft und Praxis verschiedene Modelle diskutiert. Im Bereich der Verkehrsinfrastruktur sind derzeit insbesondere das A-Modell, das F-Modell und das V-Modell (Verfügbarkeitsmodell) relevant (Böger, 2014). Diese jeweils spezifischen Lösungsansätze lassen sich in die generelle ökonomische Klassifikation von Finanzierungs-, Betreiber-, Konzessions- und Kooperationsmodellen einordnen (Bundesverband deutscher Banken, 2004).

Die begrifflichen Differenzierungen beziehen sich darauf, welche der Aufgaben im Lebenszyklus eines PPP-Objektes in welcher Art und Weise von Privaten übernommen wird:

- Finanzierungsmodelle übertragen nur die Finanzierungsfunktion an Private;

- größere Vorteile verspricht man sich von Betreibermodellen, bei denen ein privater Akteur ganz oder teilweise Finanzierung, Bau und Betrieb einer Infrastruktur übernimmt.

- Bei Konzessionsmodellen wird dagegen dem privaten Konzessionär im Vergleich zum Betreibermodell zusätzlich das Recht eingeräumt, sich über direkte Nutzergebühren zu refinanzieren.

Als Konzessionsmodell ausgestaltet sind die sogenannten F-Modelle: bei diesem Ansatz wird eine Infrastruktur von einem Konzessionär aus- bzw. neu gebaut und über 30 Jahre betrieben und erhalten, bevor sie an den Staat zurückfällt (BOT Built-Operate-Transfer). Die Finanzierung erfolgt über eine vom Konzessionär erhobene Maut, die sich nach den Grundsätzen des europäischen Gebührenrechts an den durchschnittlichen Infrastrukturkosten $\mathrm{zu}$ orientieren hat. Das 
F-Modell wird aufgrund der schlechten Erfahrungen mit den beiden realisierten Pilotprojekten (Warnowquerung Rostock und Herrentunnel Lübeck) in Deutschland derzeit nicht mehr verfolgt (Knorr/Eisenkopf, 2008).

Das so genannte A-Modell wird in Deutschland seit dem Jahr 2007 praktiziert. Es ist am Grundgedanken des Betreibermodells orientiert, da der private Betreiber nicht selbst eine Maut erhebt. Bei dieser Lösung übernimmt ein in einem wettbewerblichen Verfahren ausgewählter privater Betreiber bzw. ein Betreiberkonsortium den Ausbau, Betrieb und den Erhalt eines bestehenden Autobahnabschnitts über 30 Jahre. Der private Betreiber refinanziert sich aus dem Gebührenaufkommen der Lkw-Maut für den betreffenden Streckenabschnitt, welches der Bund vereinnahmt und an ihn weiterleitet bzw. einer auslastungsunabhängigen Schattenmaut (seit der 2. Projektstaffel 2011). Außerdem wird zum Teil eine Anschubfinanzierung seitens der öffentlichen Hand geleistet, deren Höhe in einem wettbewerblichen Verfahren ermittelt wird.

Die erste Staffel der A-Modelle mit 4 Pilotprojekten wurde mittlerweile realisiert und auch bereits evaluiert. Eine zweite Staffel mit 8 definierten Projekten startete 2011. Im Zuge der Weiterentwicklung der Finanzierungsmechanismen wurde auch erstmals das Verfügbarkeitsmodell (V-Modell) angewandt. Bei dieser Beschaffungsvariante erhält der Betreiber ein verkehrsmengenunabhängiges Verfügbarkeitsentgelt, das sich nach Umfang und Qualität der Verfügbarkeit eines bestimmten Streckenabschnittes für die Verkehrsteilnehmer bemisst, einschließlich entsprechender Bonus-Malus-Regelungen. Der entscheidende Unterschied zwischen dem A- und dem V-Modell besteht also darin, dass der Betreiber beim V-Modell kein direktes Verkehrsmengenrisiko trägt.

Ende April 2015 haben das BMVI und das BMF eine neue Generation von PPPProjekten angekündigt, bei denen die Finanzierung der Verkehrsinfrastruktur neugestaltet, die Vergütungsmechanismen optimiert und damit der Anwendungsbereich von öffentlich-privaten Partnerschaften erweitert werden soll. Die 11 avisierten Projekte arbeiten ausschließlich mit dem Verfügbarkeitsmodell. Private Kapitalgeber sollen (z. B. über eine Anleihenfinanzierung) sehr viel stärker in die Projekte eingebunden werden und ggfls. sogar deren Federführung übernehmen können. Die neuen PPP-Projekte wurden auch im neuen Bundesverkehrswegeplan explizit erwähnt. 


\section{Gesamtwirtschaftlicher Nutzen von PPP- Projekten}

\section{PPP als Beschaffungsvariante}

Die Vorteilhaftigkeit von PPP-Lösungen für Verkehrsinfrastrukturprojekte wird in der verkehrspolitischen Diskussion unterschiedlich bewertet. Während private Interessengruppen (Bauwirtschaft, Finanzdienstleister) und Teile der Politik PPP als Win-Win-Situation für die Gesellschaft oder sogar als Investmentchance präsentieren, beklagen Kritiker die Unwirtschaftlichkeit von PPP-Lösungen und insbesondere die Intransparenz aufgrund der Geheimhaltung der Verträge (Beckers/Hirschhausen/Klatt, 2005; Beckers et. al., 2011).

Ein grundsätzliches Problem von PPP besteht in der im Begriff der öffentlichprivaten Partnerschaft angelegten Annahme der Interessenharmonie zwischen den beteiligten Akteuren. Zwar setzt das Zustandekommen jedweder wirtschaftlicher Transaktion das wechselseitige Interesse der Akteure an derselben voraus. Hieraus eine generelle Übereinstimmung der Ziele von öffentlichen und privaten Akteuren in einer PPP abzuleiten, erscheint aber gewagt. Aus dem öffentlichen Interesse an einer möglichst effizienten Aufgabenerfüllung einerseits und dem Gewinnmaximierungskalkül privater Unternehmen andererseits erwächst vielmehr ein grundlegender Zielkonflikt zwischen den Parteien. Es bedarf daher umfangreicher und ausgefeilter institutioneller Regelungen, um diesen Konflikt produktiv zu handhaben und zu effizienten Kooperationslösungen zu kommen (Eisenkopf/Schnöbel, 2007).

Ein wesentliches Problem der Leistungsbeziehung zwischen öffentlichen und privaten Partnern liegt in der langfristigen Bindung der Betroffenen in Verbindung mit der hohen Komplexität der Transaktionen und den Informationsasymmetrien zwischen den Parteien begründet. Diese Konstellation führt dazu, dass sich in den zugrundeliegenden Kooperationsverträgen nicht alle relevanten Aspekte berücksichtigen lassen und erheblicher Raum für Nachverhandlungen besteht. Da die Verträge nicht abschließend formuliert werden können, hat ein opportunistischer Vertragspartner bei (exogenen) Veränderungen der Rahmenbedingungen einen Anreiz zu Nachverhandlungen. Grundsätzlich besteht dieser Anreiz für beide Vertragsparteien. So sind nach Fertigstellung des in der Regel sehr spezifischen Infrastrukturvorhabens, das kaum einer anderen Verwendung zugeführt werden kann, sowohl die öffentliche Hand als auch der private Betreiber dem Opportunismus der Gegenseite ausgesetzt, wenn ein Störereignis auftritt (z. B. Auslastungsschwierigkeiten aufgrund exogener Schocks, unerwartete Kostensteigerungen). Eine solche Problematik ergibt sich insbesondere 
am Ende der Laufzeit im Hinblick auf die Rückführung der Infrastruktur bzw. deren Restwert.

Politökonomische Überlegungen legen nahe, dass insbesondere der private Investor bei einer PPP dieses sogenannte hold-up-Problem zu seinen Gunsten ausnutzen wird. Da es letztlich um eine Daseinsvorsorgeaufgabe geht, für die der Staat gegenüber den Bürgern Verantwortung trägt, verfügt der private Betreiber der Infrastruktur über eine stärkere Verhandlungsmacht und kann sich Effizienzgewinne besser aneignen bzw. bei Leistungsstörungen zu seinen Gunsten nachverhandeln.

Dieses Problem wird dadurch verstärkt, dass zwangsläufig Informationsasymmetrien zwischen öffentlicher Hand und privatem Akteur bestehen. Man spricht in diesem Zusammenhang von einer Principal-Agent-Beziehung zwischen öffentlicher Hand und privatem Investor. Der öffentliche Auftraggeber kann insbesondere häufig nicht entscheiden, ob Leistungsstörungen auf Versagen des Agenten (privater Infrastrukturbetreiber) oder auf exogene Einflüsse zurückzuführen sind, die dieser nicht zu vertreten hat. Hieraus resultieren $u$. U. erhebliche Überwachungs- und Kontrollkosten (Agency Costs). Diese entstehen insbesondere bei der Durchsetzung vertraglicher Ansprüche während der Laufzeit und bei der Abwicklung des Vertrages.

\section{Effizienz von PPP - Mustervoraussagen zu Vor- und Nachteilen}

Eine zentrale Frage bei der Beurteilung von PPP als drittem Weg zwischen der öffentlichen und privaten Realisierung von Daseinsvorsorgeaufgaben ist die (behauptete) Überlegenheit hinsichtlich der Effizienz. Wirtschaftlichkeitsrechnungen, welche die Effizienz von PPP mit anderen Beschaffungsalternativen vergleichen, beziehen sich in der Regel auf Kriterienkataloge, die als Public Sector Comparator (PSC) v. a. in Großbritannien entwickelt wurden.

Wichtig ist, dass bei solchen Rechenansätzen die Vorteilhaftigkeit von PPP immer unter dem Aspekt einer zugegebenermaßen komplexen Lebenszyklusbetrachtung analysiert wird. Die wichtigsten hierbei zu betrachtenden Kostenarten sind:

- Investitionskosten: Hier besteht die begründete Vermutung, dass mittels PPP insbesondere durch die enge Verzahnung von Projektierung, Planung, Erstellung und Erhalt in einer Hand Kosteneinsparungen realisiert werden können (Beckers/ Hirschhausen/Klatt, 2005).

- Finanzierungskosten: Privaten wird zwar allgemein die Fähigkeit zu einem effizienten Finanzmanagement zugeschrieben, doch kann die öffentliche 
Hand auf merklich günstigere Konditionen bei der Aufnahme von Kapital zurückgreifen. Aufgrund der Cashflow-Struktur bei großen Infrastrukturprojekten (hohe Anfangsauszahlung, relativ geringe periodische Cashflows, lange Amortisationszeiten) müssen private Investoren anders als die öffentliche Hand Risikoaufschläge bei der Ermittlung der Kapitalkosten kalkulieren. Andererseits kommt der Kapitalbereitstellung durch private Akteure eine Haftungsfunktion zu, die eine effiziente Ressourcenbewirtschaftung fördert und Risiken absichert.

- Betriebs- und Unterhaltungskosten: Vorteile privatwirtschaftlicher Aktivitäten können vor allem in effizienteren Organisationsmodellen für die Bewirtschaftung und Instandhaltung sowie in geringeren Personalkosten liegen.

- Transaktionskosten und Agency Costs: Mit der Umsetzung eines Projekts in Form von PPP sind Transaktionskosten und Agency Costs verknüpft (insbesondere Kosten für Verhandlungen, Nachverhandlungen, Auseinandersetzungen bei Leistungsstörungen oder über den Restwert einer Infrastruktur). Transaktionskosten und Agency Costs von PPP wurden bisher im Regelfall nicht transparent gemacht, sind aber gleichwohl ökonomisch relevant.

Im Hinblick auf die Gesamtwirtschaftlichkeit von PPP-Projekten sind grundsätzlich keine allgemeingültigen Aussagen möglich; ein Wirtschaftlichkeitsvergleich ist immer einer Einzelfallbetrachtung vorbehalten, deren Ergebnis von den konkreten Rahmenbedingungen des Projekts abhängt. In der Vergangenheit bestand allerdings aus ökonomischer Sicht Anlass zu der „Mustervoraussage“, dass bei konventioneller Realisierung durch den Staat Kostensenkungspotentiale nicht (ausreichend) ausgeschöpft wurden.

Wie noch ausgeführt werden wird, ist die ökonomische Erfolgsbilanz der ersten Fernstraßen-PPP in Deutschland allerdings durchaus umstritten. Hinzuweisen ist auch auf die Transaktionskosten und Agency Costs, die bei Effizienzvergleichen in der Regel nicht berücksichtig werden, da sie einer Quantifizierung ex ante kaum zugänglich sind. Daher besteht die Gefahr, dass produktive Effizienzvorteile durch höhere Transaktionskosten oder Agency Costs kompensiert bzw. sogar überkompensiert werden, so dass PPP für die Gesellschaft insgesamt zum Zuschussgeschäft werden (Mühlenkamp, 2004). 


\section{Erfahrungen mit PPP-Projekten in Deutschland}

PPP-Projekte sind vor ihrer Realisierung ausgehend von $\S 7$ Bundeshaushaltsordnung (BHO) grundsätzlich einer Wirtschaftlichkeitsuntersuchung zu unterziehen; dabei ist die Vorteilhaftigkeit dieser Beschaffungsvariante nachzuweisen. Erweist sich die PPP-Variante als vorteilhaft gegenüber der konventionellen Beschaffung, kann das Projekt ausgeschrieben werden. Vor der Vergabe ist es aber einer abschließenden Wirtschaftlichkeitsrechnung zu unterziehen. Hinzu tritt ein Projektcontrolling während der Durchführung und nach Abschluss des Projektes (Bundesrechnungshof, 2013).

Trotz dieser umfangreichen vorzunehmenden Wirtschaftlichkeitsprüfungen ist nach wie vor umstritten, ob PPP im Bereich der Straßeninfrastruktur zu Effizienzsteigerungen beitragen oder eher unwirtschaftlich sind. Die Diskussion darüber entzündet sich insbesondere an einem Prüfbericht des Bundesrechnungshofes (BRH) zur Wirtschaftlichkeit von öffentlich-privaten Partnerschaften aus dem Jahr 2014. In diesem Bericht werden die seitens des Bundesverkehrsministeriums vorgenommenen Wirtschaftlichkeitsuntersuchungen heftig kritisiert und kumulierte Mehrkosten der PPP-Beschaffungsvariante von 1,9 Mrd. Euro über alle fünf betrachteten Projekte kalkuliert. Während das Bundesverkehrsministerium im Kostenvergleich Einsparungen von 1,6 \% bis 39,5 \% bei den PPP-Lösungen ermittelt und es nach seinen Berechnungen nur bei der Maßnahmen A8 Ulm/Elchingen-Augsburg/West zu um ca. $10 \%$ höheren Kosten kommt, ermittelt der BRH Mehrkosten der jeweiligen Projekte von 7,9 \% bis $46 \%$ (Bundesrechnungshof, 2014).

Inhaltlich argumentiert der Rechnungshof, dass die Kosten der konventionellen Bereitstellung im Vergleich zur PPP-Variante vom Bundesverkehrsministerium regelmäßig und systematisch zu hoch ausgewiesen würden. Die Verkehrs- und Mauteinnahmeprognosen und damit die Zahlungen an die privaten Betreiber würden in der Wirtschaftlichkeitsrechnung im Gegenzug zu niedrig angesetzt. Auch die Einbeziehung zusätzlicher Nutzen der PPP-Variante (insbesondere wegen früherer Verfügbarkeit der Infrastruktur) sei unsachgemäß und benachteilige die konventionelle Beschaffungsvariante. Außerdem würden Anrechnungsmodalitäten von PPP-Projekten auf die Verteilung von Straßenbaumitteln nach Länderquoten bei den Ländern Anreize schaffen, Straßenbauprojekte als PPP umzusetzen, da sie so zusätzliche Mittel jenseits der Länderquote erhalten könnten (Bundesrechnungshof, 2014).

Ein zentrales Problem bei der abschließenden Würdigung dieser Befunde stellt die mangelnde Transparenz und Nachvollziehbarkeit der jeweiligen Rechnungen dar, da weder die Wirtschaftlichkeitsrechnungen des Bundesverkehrsministeriums noch die des Bundesrechnungshofs der Fachöffentlichkeit im 
Detail offengelegt werden. Es fällt daher schwer, ein abschließendes Bild über die tatsächliche Wirtschaftlichkeit von streckenbezogenen PPP im Bereich der Bundesfernstraßen zu gewinnen.

Generell erscheint eine kritische Sicht auf die Wirtschaftlichkeit der PPPProjekte durchaus angebracht. Es dürfte realistisch sein, dass die Finanzierungskonditionen für private Betreiber in jedem Fall schlechter ausfallen sollten als bei konventioneller Erstellung. Damit ist auch klar, dass das PPP-Konzept sich insbesondere als Beschaffungsvariante über Kosteneinsparungen infolge des Lebenszyklusansatzes bei Planung, Bau, Betrieb und Erhalt qualifizieren muss. Außerdem ist auf die nicht in den Rechenwerken erfassten Transaktionskosten und Agency Costs hinzuweisen, die vor, während und zum Abschluss der Projektdurchführung auftreten können.

Mühlen auf das Wasser der PPP-Kritiker dürften die jüngsten Berichte über wirtschaftliche Schwierigkeiten bei fast allen A-Modellen aus der ersten Vergabestaffel darstellen. Es wird kolportiert, dass sich die hohen wirtschaftlichen Erwartungen der Investoren bei keinem der A-Modelle erfüllt haben. Als verantwortlich hierfür wird insbesondere die hinter den Erwartungen zurückgebliebene und -bleibende Entwicklung der LKW-Mauteinnahmen gesehen. Die kritische Entwicklung bei A1-Mobil wird explizit auf die von den ursprünglich prognostizierten Wachstumsraten des Lkw-Verkehrs massiv nach unten abweichende Verkehrsentwicklung zurückgeführt. Neben den zahlreichen Schiedsgerichtsverfahren hat in diesem Fall erstmals ein Konsortium den Bund verklagt. Dies illustriert überdeutlich die hohe Relevanz der erwähnten Agency Costs und die Probleme bei der Risikoallokation in solchen komplexen Projekten.

\section{Fazit: Brauchen wir mehr PPP im Bundesfernstraßennetz?}

In der Summe führen die Überlegungen zu dem sogar vom Bundesrechnungshof durchaus anerkannten Argument, dass PPP neue Impulse für die Straßenbauverwaltung generieren können und daher zu organisationalem Lernen beitragen. Hinsichtlich der gesamtwirtschaftlichen Nutzen-Kosten-Bilanz der PPP-Projekte scheint jedoch Vorsicht angebracht. Weitere PPP-Projekte sollten daher nur auf den Weg gebracht, wenn die Vorbehalte in Hinblick auf die Wirtschaftlichkeit solcher Lösungen durch unabhängige Untersuchungen überzeugend ausgeräumt wurden. Sie sollten dann ausschließlich als Verfügbarkeitsmodelle konzipiert werden, um Auslastungsrisiken zu vermeiden und einer strikten Evaluation in allen Projektphasen unterliegen.

Es sollte allerdings klar sein, dass PPP-Lösungen ausschließlich als Beschaffungsvariante zur Kompetenz- und Wissensgenerierung zum Einsatz kom- 
men sollten, um z. B. Benchmarks für Prozesse in den öffentlichen Beschaffungsinstitutionen $\mathrm{zu}$ entwickeln. Öffentlich-private-Partnerschaften bei Verkehrsinfrastrukturprojekten sind für sich genommen nicht in der Lage, die Instandhaltungs- und Bereitstellungskrise der bundesdeutschen Infrastrukturpolitik zu überwinden. In einem komplexen Fernstraßennetz verursachen sie immer erhebliche Abstimmungs- und Integrationsprobleme.

Dies gilt umso mehr in einer durch die vorgesehene Einrichtung einer Infrastrukturgesellschaft für Planung, Bau, Betrieb, Erhaltung und Finanzierung der Bundesautobahnen und ggfls. auch von Bundesstraßen veränderten Governance der Infrastrukturbereitstellung. Mit dieser Weichenstellung für eine institutionelle Reform sollen ja gerade im System selbst Anreize für mehr Effizienz bei der Bereitstellung von Bundesfernstraßen geschaffen werden. PPP stellen aber im Rahmen der Governance einer solchen Infrastrukturgesellschaft einen Fremdkörper dar, denn die Infrastrukturgesellschaft selbst will ja im Kern die effiziente Infrastrukturbereitstellung aus einer Hand im Sinne einer Lebenszyklusbetrachtung realisieren. Sinn und Zweck weiterer PPP-Projekte wäre dann primär die Attrahierung privaten Kapitals.

Die Gesamtwirtschaftlichkeit vom PPP wird jedoch aufgrund der höheren Finanzierungskosten Privater bereits bei streckenbezogenen Lösungen häufig in Frage gestellt. Umso mehr bestehen Bedenken bezüglich privat finanzierter PPPVorhaben, die sich auf Teilnetze beziehen und damit z. B. den Autobahnkonzessionsgesellschaften in Frankreich ähnlich wären. Erfreulicherweise wurden daher im Gesetzgebungsverfahren zur Infrastrukturgesellschaft weitreichende Privatisierungsschranken gegen die Umsetzung von Teilnetz-PPP gesetzt; die Verkehrspolitik sollte auch bei PPP-Einzelprojekten in Zukunft äußerste Zurückhaltung walten lassen.

\section{Literatur}

Arbeitsgruppe zum Thema „Wirtschaftlichkeitsuntersuchungen bei PPP-Projekten“

(Arbeitsgruppe) (2006), Leitfaden „Wirtschaftlichkeitsuntersuchungen bei PPP-Projekten“, Berlin.

Beckers, T., C. von Hirschhausen und J. P. Klatt (2005), Aktuelle ÖPP-Modelle für die Bundesfernstraßen - Eine ökonomische Analyse, München.

Beckers, T., J. P. Klatt, J. Kühling und A. Bäuml (2011), Institutionelle Lösungen für die Bundesfernstraßenfinanzierung: Eine Analyse aus ökonomischer und juristischer Perspektive, Studie im Auftrag des ADAC e. V., München.

Böger, T. (2014), PPP projects in Germany. Konference om OPP med fokus på transportsektoren, 27th May, Hellerup. 
Bundesministerium für Verkehr und digitale Infrastruktur (BMVI) (2016), Bundesverkehrswegeplan 2030, August, Berlin.

Bundesrechnungshof (2013), Gutachten des Beauftragten für Wirtschaftlichkeit in der Verwaltung zu Wirtschaftlichkeitsuntersuchungen bei Öffentlich Privaten Partnerschaften (ÖPP) im Bundesfernstraßenbau, Bonn.

Bundesrechnungshof (2014), Bericht an den Haushaltsausschuss des Deutschen Bundestages nach § 88 Abs. 2 BHO über Öffentlich Private Partnerschaften (ÖPP) als Beschaffungsvariante im Bundesfernstraßenbau, Bonn.

Bundesverband deutscher Banken (2004), Public Private Partnership - Chance für die Modernisierung von Infrastruktur und Verwaltung. Reihe Daten, Fakten, Argumente, Berlin.

Delmon, J. (2011), Public-Private Partnership Projects in Infrastructure. An Essential Guide for Policy Makers, Cambridge.

Eisenkopf, A. und C. Schnöbel (2007), „Die Zukunft des öffentlichen Infrastrukturauftrags im Verkehrssektor: Chancen und Risiken von Public Private Partnerships“, in: Jansen, Stephan A., Birger P. Priddat und Nico Stehr (Hrsg.): Die Zukunft des Öffentlichen. Multidisziplinäre Perspektiven für eine Öffnung der Diskussion über das Öffentliche, Wiesbaden, S. 49-83.

Knorr, A. und A. Eisenkopf (2008), „Road Infrastructure PPPs in Germany. Why did the F-Model fail? - Two Case Studies“, FÖV Discussion Papers 45, German Research Institute for Public Administration, Speyer.

Kommission „Verkehrsinfrastrukturfinanzierung“ (2000), Schlussbericht, Berlin, 05.09.

Kommission „Zukunft der Verkehrsinfrastrukturfinanzierung“ (2012), Bericht Dezember 2012, Berlin.

Mühlenkamp, H. (2004), „Public Private Partnership“ ökonomisch analysiert - Eine Abhandlung aus der Sicht der Transaktionskostenökonomik und der neuen Politischen Ökonomie, Speyerer Arbeitsheft Nr. 166, Speyer. 